\title{
Set Theory
}

We will present the most important elements of set theory, because without appropriate knowledge one cannot acquire a sufficient understanding of Brownian motion. Set theory is also needed when it comes to the theory of random variables, probability theory, information economics, or game theory. Since set theory is not dealt with in sufficient detail in formal training of economists, we will discuss the required issues here.

\subsection{Notation and Set Operations}

Term of Set A set is a collection of various objects. If you want to describe a set you have to specify its elements. This happens in curly brackets where the elements are shown following a colon or a vertical line. The set

$$
M:=\left\{x \in \mathbb{R} \mid a x+b x^{2} \geq 0\right\}
$$

contains those real numbers $x$ that satisfy the inequality $a x+b x^{2} \geq 0$. If a set contains only a single element, it is called a point set.

If the numbers are real, one likes to use abbreviations in notation. The set $A$ of numbers greater than 0 and less than 1 should actually be written in the form

$$
A:=\{x \in \mathbb{R} \mid 0<x<1\}
$$

which is quite cumbersome. Instead, the more compact notation

$$
A:=(0,1)
$$


is used. This applies also to half-open and closed intervals.

The set of real numbers is denoted by $\mathbb{R}$, the set of natural numbers by $\mathbb{N}$, the set of integers by $\mathbb{Z}$, and the set of rational numbers by $\mathbb{Q} .^{1}$ The empty set $\varnothing$ contains no elements.

The elements are grouped together in a set, regardless of their sequence. So if $\Omega$ consists of the two elements $u$ and $d$, it does not matter whether $\Omega=\{u, d\}$ or $\Omega=\{d, u\}$ is written. In some economic applications, however, the order of the elements is important. One speaks then of a "pair" and writes $(u, d)$ if the sequence of elements is relevant. There can also be more than two entries such as $(u, d, d, u)$. In this case one speaks of a "tuple." If these pairs or tuples are combined into a set, this set is no longer $\Omega$ but a new set. Depending on the length of the tuple this new set is called $\Omega^{2}$ for two entries in a pair and $\Omega^{T}$ for $T$ entries in the tuple. ${ }^{2}$

Set Operations You can unite sets and you can calculate their intersection and difference. Considering two sets this means the following.

The union contains all their elements. The symbol $\cup$ is used to identify the union. For example, the following applies

$$
\{1,2\}=\{1\} \cup\{2\}
$$

The intersection contains elements found in both sets. The symbol $\cap$ indicates intersection. For example, the following is true

$$
\emptyset=\{1\} \cap\{2\} .
$$

Sets whose intersection is empty are called disjoint.

Let us focus on a set $A$. We denote this a subset of $B$ if it only contains elements from $B$ regardless whether these are all or only some elements. It is in short $A \subset B$. Then the following always applies

$$
A \subset B \quad \Longrightarrow \quad A \cup B=B \quad \text { and } \quad A \cap B=A \text {. }
$$

Each of the last two relationships characterizes subsets.

The difference $A \backslash B$ of two sets is the set containing all elements from $A$ that are not in $B$. For the difference rules of calculation apply which are similar to arithmetics. ${ }^{3}$ When $A$ and $B$ are subsets of a set $C$, then the following

\footnotetext{
${ }^{1}$ While there exist different opinions about whether zero is a natural number, we assume it is. Rational numbers are regularly defined as quotients of integers $x=\frac{m}{n}$, where $m, n \in \mathbb{Z}$ and $n \neq 0$. ${ }^{2}$ For the sake of completeness, it should be noted that the representation $\Omega \otimes \Omega=\Omega^{2}$ is used for pair formation of two different sets. The mathematically trained reader then knows that the new set consists of the ordered pairs $(u, d)$ where $u \in \Omega$ and $d \in \Omega$.

${ }^{3}$ The rules are not always similar. A simple rule says that if there is a $\backslash$ difference, the symbols $\cap$ and $\cup$ are swapped.
} 
Fig. 2.1 Intersection of sets $A$ and $B$

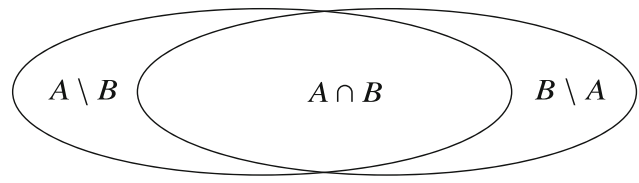

Fig. 2.2 The difference between $A \backslash B \cap C$ (left) and $A \backslash(B \cap C)$ (right)

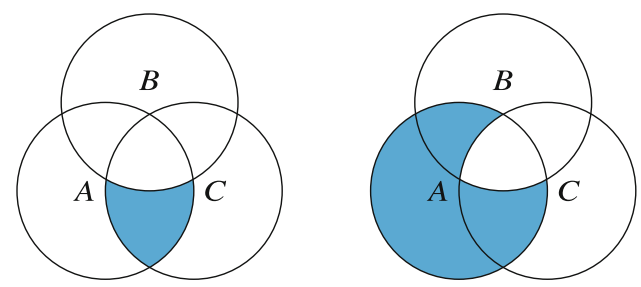

is valid:

$$
\begin{aligned}
C \backslash(A \cap B) & =(C \backslash A) \cup(C \backslash B) \\
C \backslash(A \cup B) & =(C \backslash A) \cap(C \backslash B) \\
C \backslash(C \backslash A) & =A .
\end{aligned}
$$

One can convince oneself of the correctness of these rules with the help of the socalled Venn diagrams ${ }^{4}$; we are using them here without further explanation. A Venn diagram is a simple symbolic representation in which the sets are always indicated by circles or ellipses. The drawing illustrates the statements on union, intersection, and difference, see Fig. 2.1.

The graph shows, for example, that the set $A \cap B$ (the inner part of both ellipses) represents a subset of $A \cup B$ (the totality of both ellipses), thus $A \cap B \subset A \cup B$.

Computation Rules The following rule applies to set operations and is based on the rules of arithmetic: line operation takes precedence over union and intersection. As a result, brackets that include a difference can be omitted. For example, one writes

$$
(A \backslash B) \cap C \quad \text { shorter } \quad A \backslash B \cap C .
$$

We know that $1 / 2+3$ is something other than $1 /(2+3)$. A similar case can be found in Fig. 2.2. There are two terms that differ only in the brackets: $A \backslash B \cap C$ and the set $A \backslash(B \cap C)$. The second set differs from the first in a small but not negligible part: it contains those elements of $A$ which are not in $C$.

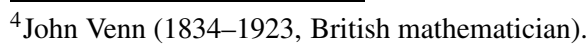


If the difference $B \backslash A$ is formed, where $B=\Omega$ represents the initial set of all elements, the result is also called complement of $A$ and one simply writes $A^{c}$.

Often set operations are identified with corresponding calculation rules from arithmetic: the difference "looks like" subtraction, while the union reminds one of addition. Note, however, that there are some calculation rules that are in clear contrast to arithmetic. For example, the following applies to all $A$ :

$$
A \cap A=A \quad \text { and } \quad A \cup A=A .
$$

There is no equivalent in the arithmetic of real numbers.

An Exercise We illustrate the calculation rules described using two equations. For this purpose, we represent a union $A \cup B$ of two sets by two new sets such that the two new sets are disjoint. This is relatively easy because

$$
A \cup B=(A \backslash B) \cup B
$$

must be fulfilled. Let us first realize that the union on the right is indeed identical to $A \cup B$; second, these two new sets are disjoint. The second condition is obviously met, because $A \backslash B$ by definition only contains elements that are not included in $B$, i.e.,

$$
A \backslash B \cap B=\emptyset .
$$

If the union of the two sets is to be determined precisely, the procedure would be as follows:

$$
\begin{aligned}
A \backslash B \cup B & =\{x \mid x \in A \backslash B \text { or } x \in B\} \\
& =\{x \mid(x \in A \text { and } x \notin B) \text { or } x \in B\} \\
& =\{x \mid x \in A \text { or } x \in B\} \\
& =A \cup B .
\end{aligned}
$$

The Venn diagram in Fig. 2.3 illustrates our considerations. Similarly, it is clear that for any set $A$ and $B$

$$
A=(A \backslash B) \cup(A \cap B)
$$

applies. 

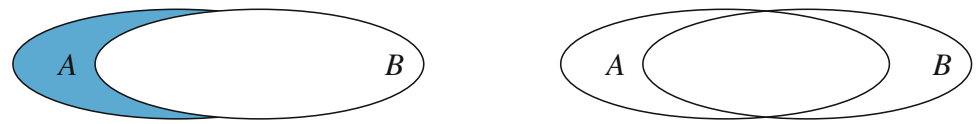

Fig. 2.3 The union of the disjoint sets $A \backslash B$ (left, blue) and $B$ results in $A \cup B$ (right)

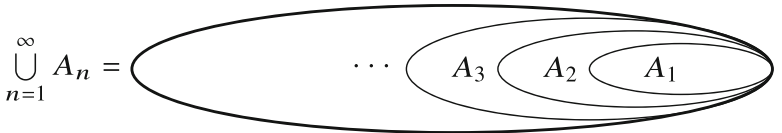

Fig. 2.4 An infinite union of ascending subsets in the Venn diagram

At times we have to deal with infinite operations of unions and intersections. Let us assume that an infinite sequence of sets $A_{1}, A_{2}, \ldots$ exists. ${ }^{5}$ The infinite union

$$
\bigcup_{n=1}^{\infty} A_{n}
$$

is the set that contains all elements from each set $A_{n}$. Figure 2.4 illustrates such an infinite union. Likewise, the intersection

$$
\bigcap_{n=1}^{\infty} A_{n}
$$

is the set which contains only those elements existing in all sets $A_{n}$.

As an example

$$
\mathbb{N}=\bigcup_{n=0}^{\infty}\{n\}
$$

applies for the set of natural numbers because the infinite union includes all natural numbers. Likewise we have

$$
\emptyset=\bigcap_{n=0}^{\infty}[n, \infty)
$$

since an element that should be in all half-open intervals $[n, \infty)$ must be greater than any natural number $n$-and such a number does not exist. ${ }^{6}$

\footnotetext{
${ }^{5}$ Such a sequence is often written as $\left(A_{n}\right)_{n=1, \ldots}$.

${ }^{6}$ The object $\infty$ is not a number, because you cannot use it in calculations. For example, $1+\infty=\infty$, from which $1=0$ would follow if $\infty$ were a natural number.
} 
Another example of real numbers is a good illustration of the concept. As $A_{n}$ we choose the interval $A_{n}:=\left[\frac{1}{n}, 1-\frac{1}{n}\right]$ with $n>1$. If $n$ increases the interval also increases which is why $A_{n}$ is a subset of $A_{n+1} \cdot\left(A_{n}\right)_{n=1, \ldots}$ is indeed a sequence of subsets. The limit of this sequence is then the interval

$$
\bigcup_{n=1}^{\infty}\left[\frac{1}{n}, 1-\frac{1}{n}\right]=(0,1),
$$

because each number in the open interval $(0,1)$ lies (for sufficiently large $n$ ) in a set $A_{n}$. Further, the boundary values 0 and 1 lie neither in the open interval $(0,1)$ nor in one of the sets $A_{n}$. We hope that both examples help to understand what is meant by an infinite sequence of sets.

Power Set The set of all subsets of a set $\Omega$ is called the power set which is denoted by $\mathcal{P}(\Omega)$. One has to realize that the power set is much larger than the set itself.

Think about a situation with six elements $\Omega=\{1,2,3,4,5,6\}$. If we look at all subsets of this finite event space, we arrive at a total of $2^{6}=64$ subsets of the event space, namely ${ }^{7}$

$$
\begin{aligned}
\mathcal{P}(\Omega)=\{\emptyset,\{1\},\{2\}, \ldots,\{6\},\{1,2\},\{1,3\},\{1,4\}, \ldots, \\
\{1,2,3\},\{1,2,4\}, \ldots,\{1,2,3,4,5,6\}\} .
\end{aligned}
$$

Also for infinite sets, the power set is much larger than the initial set. This is a bit surprising, because it is not clear, why one can distinguish different "levels" (more precisely cardinalities) of infinity. We have put these considerations in Sect. 7.1. ${ }^{8}$

\subsection{Events and Sets}

In colloquial language it is said that "events occur." But what is an event? Specific examples are a dice roll, the share price at the end of a trading day, or the move of a chess player. In economic contexts, an event often determines an economic result (such as a pay-out, a pay-in, a profit).

However, an economist is usually not satisfied with the statement that this or that event could occur. Rather, economists calculate the expected values or variances of payments triggered by those events. In order to do so, mathematicians operate with the term "set." To understand this, let us take a closer look at the example of a dice.

\footnotetext{
${ }^{7}$ There is a total of $\left(\begin{array}{c}6 \\ n\end{array}\right)$ subsets that contain exactly $n$ elements. If we add these binomial coefficients over all $n$, we get the result because $\sum_{n=0}^{6}\left(\begin{array}{l}6 \\ n\end{array}\right)=64$.

${ }^{8}$ See page 103 .
} 
Dice Roll We can trust that everyone knows which characteristics an ideal dice has. If we take a closer look at several possible events related to a dice roll and identify them with certain symbols:

$\boldsymbol{A}_{1}$ The one was rolled.

$\boldsymbol{A}_{2}$ An odd score was rolled.

$\boldsymbol{A}_{3}$ The dice can no longer be found.

$\boldsymbol{A}_{4}$ The score cannot be read.

$\boldsymbol{A}_{5}$ A dice has been rolled without the score being noted.

We neither care whether the dice is thrown with the left hand or with the right hand nor whether it is pushed of the table. What matters is the score on the top. Therefore, one could describe the event of the dice roll alone by the score that appears at the end. This has the inestimable advantage that all conceivable events are completely described by six numbers. The mathematician ignores everything else.

Thus, the events $A_{3}$ and $A_{4}$ no longer differ for the mathematician: $A_{3}=A_{4}$. Since obviously no scores are given, the mathematician even writes $A_{3}=A_{4}=\emptyset$.

For the events $A_{2}$ and $A_{5}$, the actual score is not reported. However, there is something that distinguishes the two events from the events $A_{3}$ and $A_{4}$ : while $A_{3}$ and $A_{4}$ hide the scores, $A_{2}$ and $A_{5}$ do not. Here a number was definitely determined, but we were not told which it was. Mathematically, this is expressed for the last event by noting all possible scores, i.e., $A_{5}=\{1,2,3,4,5,6\}$.

Let us look at event $A_{1}$. In this event we are sure that the score was one. But then a mathematician uses the score to identify the event, i.e., $A_{1}=\{1\} .{ }^{9}$ In the same way, you can describe the event $A_{2}$ by enumerating all odd numbers. This means $A_{2}=\{1,3,5\}$.

We realize that $A_{1}$ represents a subset of $A_{2}$, that is $A_{1} \subset A_{2}$. There is a very clear interpretation for this set-theoretical representation: whenever the event $A_{1}$ occurs, the event $A_{2}$ is also true. And indeed, it is also true that the number of points is odd when a player rolls the number one. Of course, the opposite does not hold.

Elementary Event To prepare for the following chapter on measures, it is useful to introduce the terms "elementary event" and "event space."10 All these sets are specific sets.

Elementary events are those sets that have no "genuine subsets." What do we mean by this term? Since the empty set and the set itself constitute subsets, those two must not be considered. All remaining sets are the genuine ones. Elementary events therefore contain only a single element and are the smallest events which are conceivable.

\footnotetext{
${ }^{9}$ You have to distinguish this notation from $A_{1}=1$. In this case $A_{1}$ would be a real number. In the case of $A_{1}=\{1\}, A_{1}$ is a set containing only one element (the natural number 1 ).

${ }^{10}$ Anyone who is studying literature on general theory of measurement will not find these terms there. Corresponding "objects" are called differently, because one develops a theory which is not only concerned with probability measures.
} 
The event space contains all events that one wants to look at. In the following we describe the terms descriptively with the help of examples.

To get an idea of an elementary event, imagine rolling the dice once and ask yourself what scores can occur. These are 1, 2, 3, 4, 5, and 6. Since these results cannot be broken down further, we call a set an elementary event if it contains one of these numbers.

While the term is easy to understand in context of a dice roll, it is not as simple if we consider realizations of a share price: here, one must know which listings are admitted on a stock exchange and if, for example, only full Dollar quotes or quotes in jumps of 10 cents are permitted. The identification of elementary events becomes even more complicated when one thinks of the results of a parliamentary election.

Event Space We use this term to denote the set of all elementary events, commonly denoted by $\Omega$. It is either a finite or an infinite set. ${ }^{11}$

Event One does not always only want to discuss elementary events. Rather, one often wants to describe the effects that follow from a combination of several elementary events: "When rolling an odd number ..." or "At a day temperature above freezing ...." In this case we speak of composite events. Sometimes we simply use the term event. Such an event usually represents as set of elementary events. An event is thus a (arbitrary) subset of the event space, or $A \subset \Omega$. $A$ then stands for a (possibly compound) event.

Example 2.1 (Multiple Dice Rolls) Set theory also allows us to characterize somewhat more complex events. Think of games in which the dice are rolled not once but several times in a row. ${ }^{12}$ This is also easy to handle mathematically. If you have three rolls, you only have to note the three numbers in a row. Now there are two possibilities when rolling the dice several times: either the order in which the scores appear is important or it is not. If the order is relevant, the event would be described by a triple, i.e., $(2,1,2)$ with three rolls. If the order is meaningless, the mathematician would note that the obtained numbers belong to the set $\{1,2\}$.

Example 2.2 (Coin Toss, Once and Several Times) We will later look at a situation where the result will depend on the toss of a coin. Two outcomes are relevant: heads or tails. The chance of a coin standing on the edge is usually excluded as being

\footnotetext{
${ }^{11}$ In probability theory $\Omega$ is often referred to as the basic set. The elements of this set are labeled $\omega$.

${ }^{12}$ German children like to play "Mensch ärgere dich nicht!" (A literal translation is "do not be annoyed." In UK a similar game is called "Ludo." We are not aware whether this game allows the same rule as described now).

The following rule applies to this game: if a player has no meeple at all on the field (which concerns all players at the beginning of the game), he has three attempts in each round to roll the necessary number six in order to bring a meeple into play.
} 
improbable. ${ }^{13}$ Then a coin toss can be described by an element of the set $\{u, d\}$, where $u$ stands for heads and $d$ for tails. Thus the coin toss is similar to the dice roll, but here we have only two instead of six elementary events.

The situation becomes a little more complex when we look at multiple coin tosses. We will discuss details on page 24 in Example 2.4.

Example 2.3 (A Share Price) Assume that the prices of a share correspond to any real nonnegative number. The event space $\Omega$ of a share price at a future point in time thus corresponds to $\Omega=\mathbb{R}_{+} \cdot{ }^{14}$ This event space contains an infinite number of possible elementary events. Hence, the so-called power set is infinite, too. ${ }^{15}$ The power set contains all (open and closed) subintervals of real numbers as well as their unions and intersections. Such a set is extremely large.

We will use this event space again when we discuss the Lebesgue measure and the Stieltjes measure.

So far we have explained these terms using the simple examples of a dice roll, a coin toss, and a single share price. One could therefore think that the event space $\Omega$ will always have to be constructed very simply. That is by no means the case. To illustrate this point, we present more complicated examples. To do so it is necessary, however, to clarify the difference between discrete-time and continuoustime models.

Discrete-Time Models If you proceed in a discrete manner, you assume that the share price is quoted at $t=0,1, \ldots$. There are periods between these dates in which no trading takes place and, as a result, no price is determined. Whether the time periods between the dates are long (a year) or short (a minute or a second) is a technical question, but not fundamental. It is crucial that the trade is interrupted again and again. In such models it is often assumed that the price movements from a point in time to the next are also of a discrete nature with a price either rising or falling by a (fixed) percentage. In such a case, we are dealing with a discrete-time model of share price development.

While $t=0$ denotes the present, we characterize all future times with the natural numbers $t=1,2, \ldots$ up to the terminal date $T$. The terminal date can be infinite, $T \rightarrow \infty$. In this case there is "no end of the world."

\footnotetext{
${ }^{13}$ That this case can indeed occur was shown, for example, on March 24, 1965, when FC Cologne and FC Liverpool competed against each other in the quarter-finals of the European Football Cup. The three matches played between the two clubs all ended in a draw. According to the rule in existence at that time the winner had to be determined by a coin toss. When the first coin was flipped, it stopped on its edge.

${ }^{14}$ Our following considerations may be applied to the case in which the event space covers only an interval of $\mathbb{R}_{+}$.

${ }^{15}$ See page 107 for details.
} 
Example 2.4 (Binomial Model) To get a vivid idea of the discrete-time concept, we now consider a simple binomial model with a finite number $T$ future points in time, $T>1$.

Let us assume that the price of a share today is $S_{0}$. A decision-maker may use the idea that this price will change at any future time either by the factor $u(t)$ (for up) or by the factor $d(t)$ (for down) with $u(t)>d(t)>0$. The symbol $\omega_{t} \in$ $\{u(t), d(t)\}$ in this simple model means nothing else than a process that causes the previous stock price $S_{t-1}$ to change by the factor $u(t)$ or the factor $d(t)$, so that either $S_{t}=S_{t-1} \cdot u(t)$ or $S_{t}=S_{t-1} \cdot d(t)$ applies. If one has such a $\omega_{t}$ in mind, one could speak of an "elementary event of a time." However, there is no such term in the literature. If one examines all consecutive processes $\omega_{t}$ for $t=1,2, \ldots, T$, then one is dealing with a vector, and exactly such a vector is meant when the literature talks about discrete-time models of elementary events. An elementary event is therefore a vector $^{16}$

$$
\omega=\left(\omega_{1}, \omega_{2}, \ldots, \omega_{T}\right) \in \Omega^{T}
$$

The event space is then the set $\Omega^{T}$. Unlike the one-period model elementary events are no longer elements of $\Omega$ but vectors of elements of $\Omega$. Thus, events are sets of vectors. If one specifies this for the binomial model with $T=2$ future points in time, four possible elementary events can be distinguished,

$$
\omega=\left\{\begin{array}{l}
u(1), u(2) \\
u(1), d(2) \\
d(1), u(2) \\
d(1), d(2)
\end{array} .\right.
$$

In some economic examples, this model is used with an infinite time horizon. For the sake of simplicity, however, it is assumed that the factors $u$ and $d$ are constant over time. Thus, events are determined by a sequence of $u$ 's and $d$ 's. Any elementary event can be written as an infinite tuple

$$
\omega=\left(\omega_{1}, \ldots\right) \in\{u, d\}^{\infty} .
$$

Such an event space is often illustrated by a so-called binomial model. A graphical representation is used in which the entry in the event vector (i.e., a $\left.\omega_{t} \in\{u, d\}\right)$ is expressed by an upward or downward movement. Figure 2.5 represents such a model for the first three points in time. The particular path $u u d$, i.e., an elementary event is highlighted. All paths are cut off at $t=4$ and the movements continuing into infinity are only indicated. This corresponds to a coin toss with infinite repetition.

\footnotetext{
${ }^{16}$ This representation is only correct if the individual points in times all have the same $\Omega$.
} 


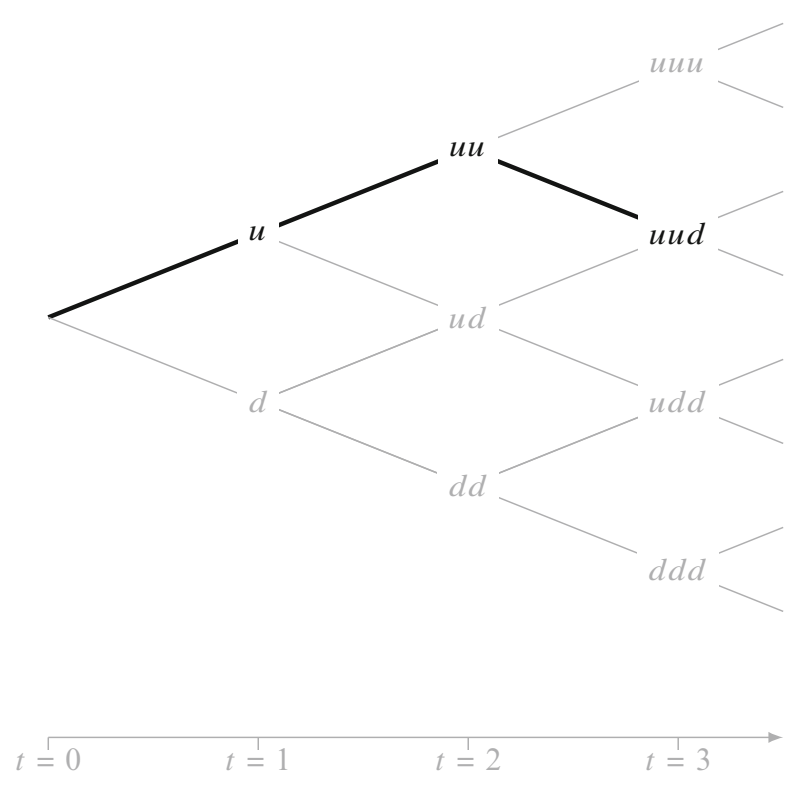

Fig. 2.5 Binomial model with events up to $t=3$, the event $u u d$ is highlighted

Our example provides further insights. Look at Fig. 2.5 and concentrate on the elementary event uud. Note that in addition to this path there exist two further elementary events $(u d u$ and $d u u)$. While their states in $t=3$ are identical, the three elementary events are not. One also speaks of a recombining binomial model.

Such models are often used in the theory of evaluating derivatives. However, even if the paths $u u d, u d u$, and $d u u$ for the underlying asset (typically a share) result in the same payment at the time $t=3$, this is not necessarily the case when valuing an option on this asset. There exist also derivatives where this value of the option at time $t=3$ depends on the path that the underlying asset has taken, while it is hard to distinguish between the elementary events $u u d, u d u$, and $d u u$ in Fig. 2.5.

Continuous-Time Models What changes when looking at a continuous-time model? A continuous model is based on the assumption that equity trading is never interrupted between two points in time. Rather, the market is trading on an ongoing basis. This implies that a stock price is given at any instant. The price is moving permanently. Furthermore, assuming that the share price can attain any value within an interval (however defined) the model is continuous in time and state.

Whether one prefers discrete or continuous models has absolutely nothing to do with the nature of reality. Rather, it is a question of usefulness.

Possible developments of a share price within a time interval $[0, T]$ can no longer be described with the help of tuples or vectors $\left(\omega_{1}, \omega_{2}, \ldots, \omega_{T}\right)$ : the number of 
entries would have to be infinite regardless of the size of the time interval. Although this "vector" does have integer column entries at any point in time, we need a column for each real number. Such an object is mathematically no longer a vector but a function.

Just as in the discrete model, an elementary event should describe the possible development of a share price over the entire time interval. If this event can be represented as a real number, then we must characterize it as a function,

$$
\omega:[0, T] \rightarrow \Omega
$$

An elementary event could be either an increasing or a decreasing development of the share price within $[0, T]$. In the first case $\omega$ would be a growing function, and in the second a decreasing one. Most elementary events will not have the property of monotonicity. Instead, one will usually observe irregular ups and downs. From now on events constitute sets of continuous functions.

Example 2.5 (Share Price Evolution) In Example 2.4, we have studied the share prices at several future dates. In this example all time indices from today $(t=0)$ to the final date $(t \rightarrow \infty)$ are available. Future share prices will then no longer be numbers but functions of time.

Then the event space $\Omega$ will get more elaborate. After all a share price evolution is a function of real numbers. We reasonably assume that this function is continuous (i.e., shows no jumps). $\Omega$ must then contain all continuous functions $f(t)$ : $[0, \infty) \rightarrow \mathbb{R}^{17}$ This set is also referred to in the literature as $C[0, \infty)$. The letter $C$ indicates "continuous."

Anyone who wants to study the Brownian motion carefully must know that there are continuous functions and differentiable functions, but they are not identical. First of all, one can prove with little effort that a differentiable function must also be continuous. But the inverse does not have to be true: continuous functions are not necessarily differentiable. Using an example of Weierstraß we show on page 107 how such functions can be constructed. The role of these functions in a Brownian motion will be discussed later.

It is not a problem to imagine single elementary events of $C[0, \infty)$. In Fig. 2.6 we have shown three conceivable share price developments. One of the shown share prices always grows at the same rate, another one fluctuates almost like a sinus function, and, finally, there is a share price development that could perhaps actually be observed on a stock exchange. Each of these functions is an elementary event from the set $C[0, \infty)$.

There is no doubt that sinusoidal or linear share price trends are highly unlikely. It is not at all clear how to define and measure probabilities of share price evolutions at this point. Although unlikely, both the linear and the sinusoidal movement cannot be

\footnotetext{
${ }^{17}$ We could exclude negative stock prices since shareholders are not liable, so $f(t):[0, \infty) \rightarrow$ $\mathbb{R}_{+}$.
} 


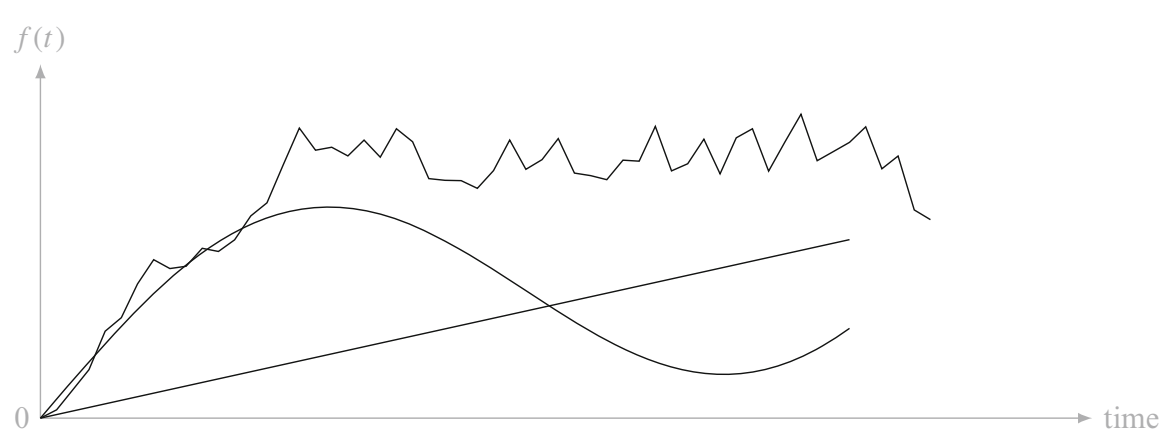

Fig. 2.6 Three elementary events in the event space $C[0, \infty)$

excluded. By contrast, no events in the sense defined here would be curves that are not continuous and show jumps. Equally unthinkable are share price developments which do not move forward in time but show a "time reversal," i.e., move back into the past. ${ }^{18}$

We would like to emphasize that all considerations are deterministic. Although uncertainty exists, we do not have probabilities yet. All examples of share price evolutions can occur. The three events mentioned (including the "random" function) assume that the future values will be described by the function $f(t)$. Probabilistic considerations will be introduced later.

The Brownian motion uses the event space $\Omega=C[0, \infty)$. Usually it is assumed that all elements of the event space start in one and the same point; for all functions $W(t) \in C[0, \infty)$ then $W(0)=a$ applies. In the figure we have chosen $a=0$, this specification will later also apply to the Brownian motions.

Figure 2.6 shows three functions being continuous and starting in the same point. These two conditions are typical for every path of the Brownian motion. However, a third characteristic of paths in Brownian motion is not recognizable in Fig. 2.6 and will be discussed later.

\footnotetext{
${ }^{18}$ Such a thing is incompatible with the concept of a function.
} 
Open Access This chapter is licensed under the terms of the Creative Commons Attribution 4.0 International License (http://creativecommons.org/licenses/by/4.0/), which permits use, sharing, adaptation, distribution and reproduction in any medium or format, as long as you give appropriate credit to the original author(s) and the source, provide a link to the Creative Commons licence and indicate if changes were made.

The images or other third party material in this chapter are included in the chapter's Creative Commons licence, unless indicated otherwise in a credit line to the material. If material is not included in the chapter's Creative Commons licence and your intended use is not permitted by statutory regulation or exceeds the permitted use, you will need to obtain permission directly from the copyright holder. 